\title{
Identification of a new relatively old star cluster in the Small Magellanic Cloud
}

\author{
Andrés E. Piatti \\ Instituto de Astronomía y Física del Espacio, CC 67, Suc. 28, 1428, Ciudad de Buenos \\ Aires, Argentina \\ e-mail: andres@iafe.uba.ar
}

Received __; accepted _ _ 


\begin{abstract}
We present results on the age and metallicity estimates of the astonishingly unstudied SMC cluster ESO 51-SC09, from CCD BVI photometry obtained at the ESO NTT with the EMMI attached. ESO 51-SC09 turns out to be a relatively small cluster $(\mathrm{FWHM}=(10 \pm 1) \mathrm{pc})$ located $\sim 4^{\circ}$ northward from the galaxy center. We report for the first time a mean cluster age of $(7.0 \pm 1.3)$ Gyr and a mean cluster metallicity of $[\mathrm{Fe} / \mathrm{H}]=(-1.00 \pm 0.15)$ dex, concluding that ESO 51SC09 belongs to the group of the oldest SMC clusters. We found that the cluster is projected onto a dominant field stellar population older (age 10-13 Gyr) and more metal-poor $([\mathrm{Fe} / \mathrm{H}]=-1.3 \pm 0.2 \mathrm{dex})$, so that the cluster could reach its current location because of its orbital motion.
\end{abstract}

Subject headings: techniques: photometric - galaxies: individual (SMC) - galaxies: star clusters. 


\section{Introduction}

As far as we are aware, ESO 51-SC09 $\left(\mathrm{RA}=00^{h} 58^{m} 57^{s} .96\right.$, Dec. $=-68^{\circ} 54^{\prime} 55^{\prime \prime} .7$, J2000) is a cataloged star cluster of the Small Magellanic Cloud (SMC, Bica et al. 2008) which has remained unstudied until the present. It is located in the outer disk of the SMC, at $\sim 4^{\circ}$ northward from the galaxy center as supposed to be at $\mathrm{RA}=00^{h} 52^{m} 45^{s}$, Dec. $=$ $72^{\circ} 49^{\prime} 43^{\prime \prime}$ (J2000) (Crowl et al. 2001). Its position should facilitate astrophysical studies, since reddening effects are at a minimum regime and the unavoidable field contamination does not represent a real constraint. On the other hand, bearing in mind the enormous interest in identifying new relatively old/old clusters in the SMC (Dias et al. 2010) and the appearance in the sky of ESO 51-SC09 like a candidate old cluster (Lauberts 1982), it is astonishing that it has not been mentioned in the literature as a valuable target. According to the hierarchical star-formation scenario found by Bonatto \& Bica (2010), the outer SMC disk would appear to be a genuine reservoir of old clusters. In fact, the oldest known cluster (NGC 121, age $=10.6 \pm 0.5$ Gyr, Dolphin et al. 2001) is also placed in the outer disk, whereas Lindsay 32 and 38 - two relatively old/old clusters (Piatti et al. 2001) - are in the ESO 51-SC09's zone, at distances smaller than $\sim 1^{\circ}$.

In this Letter we present for the first time age and metallicity estimates for ESO 51SC09. The results show that this cluster belongs to the handful of oldest clusters in the SMC (age $\geq 5$ Gyr). The impact of this finding would appear to be twofold: firstly, we actually found a new SMC cluster in the relatively old/old range. Note that different campaigns have carried out until the present searching for old star clusters in the SMC and, unfortunately, new candidates have not been identified. These results would appear not only to show that the task of finding more old star clusters in the SMC is arduous, but also it would appear a venture hardly to reach success. The amazing scarce amount of old SMC star clusters results even more noticeable when comparing it with the 456 
star clusters cataloged in the SMC (Bonatto \& Bica 2010), thus representing $\approx 1 \%$ of the SMC star cluster population. Secondly, taking into account that Piatti (2011a) predicted that we should expect to identify only one outer disk relatively old/old cluster not studied yet within those cataloged by Bica et al. (2008), and ESO 51-SC09 is uncovered to be a relatively old/old cluster, the SMC outer disk would appear not to be populated by any other unstudied old cluster.

This Letter is organized as follows: In Section 2 we describe the data collected, the reduction procedures performed, and the subsequent photometry standardization. In Section 3 we deal with the infamous cleaning process of the decontamination of field stars in the cluster Color-Magnitude Diagram, while Section 4 is devoted to the estimation of the cluster age and metallicity. Finally, Section 5 summarizes our results.

\section{Data collection and reduction}

Based on data obtained from the European Southern Observatory (ESO) Science Archive Facility under request number 33312, we collected BVI images centered on ESO 51SC09 along with observations of the SA 113 and PG 0231+51 standard fields (Landolt 1992) and calibration frames (zero, dark, sky-flat, dome-flat). The data were obtained at the ESO New Technology Telescope (NTT) with the ESO Multi-Mode Instrument (EMMI) attached $\left(\right.$ scale $=0.1665^{\prime \prime} /$ pixel $)$. We obtained for ESO 51-SC09 exposures of 600 sec per filter under seeing conditions better than $0 .^{\prime \prime} 5$, and with a mean airmass of 1.32 . For the standard fields, the exposures varied from 10 up to $100 \mathrm{sec}$, depending on the filter and the airmass (airmass range $\sim 1.1-2.2$ ).

The data reduction followed the procedures documented by the NOAO Deep Wide 
Field Survey team (Jannuzi et al. 2003) and utilized the MSCRED package in IRAF1. We processed a total of 274 images by performing overscan, trimming corrections, bias subtraction, flattened all data images, etc., once the calibration frames (zeros, darks, skyand dome- flats, etc) were properly combined. Combined dark frames obtained from 600 sec exposures showed count levels around zero. The final FOV of the images resulted to be $\sim 4.7^{\prime} \times 4.7^{\prime}$ (see Fig. 1).

Nearly 15 independent magnitude measures of standard stars from the list of Landolt (1992) were derived per filter using the APPHOT task within IRAF, in order to secure the transformation from the instrumental to the $B V I$ standard system. The standard fields PG 0231+51 and SA 113 contain between 6 and 8 standard stars each distributed over an area similar to that of the EMMI, so that we measured magnitudes of standard stars in each of its two chips. The relationships between instrumental and standard magnitudes were obtained by fitting the equations:

$$
\begin{gathered}
b=b_{1}+V+(B-V)+b_{2} \times X_{B}+b_{3} \times(B-V) \\
v=v_{1}+V+v_{2} \times X_{V}+v_{3} \times(B-V) \\
i=i_{1}+V-(V-I)+i_{2} \times X_{I}+i_{3} \times(V-I)
\end{gathered}
$$

where $a_{i}, b_{i}$ and $c_{i}(i=1,2$, and 3$)$ are the fitted coefficients, and $X$ represents the effective

\footnotetext{
${ }^{1}$ IRAF is distributed by the National Optical Astronomy Observatories, which is operated by the Association of Universities for Research in Astronomy, Inc., under contract with the National Science Foundation.
} 
airmass. Capital and lowercase letters represent standard and instrumental magnitudes, respectively. We solved the transformation equations with the FITPARAMS task in IRAF for the 2 chips simultaneously, and found mean color terms of $0.028 \pm 0.009$ in $b, 0.006 \pm 0.014$ in $v$ and $0.012 \pm 0.020$ in $i$, and mean airmass coefficients of $0.243 \pm 0.008$ in $b, 0.141 \pm 0.013$ in $v$ and $0.043 \pm 0.018$ in $i$. The rms errors from the transformation to the standard system were 0.010, 0.016 and 0.015 mag for $B, V$ and $I$, respectively, indicating an excellent photometric quality.

The stellar photometry was performed using the star-finding and point-spread-function (PSF) fitting routines in the DAOPHOT/ALLSTAR suite of programs (Stetson et al. 1990). For each image, a quadratically varying PSF was derived by fitting $\sim 40$ stars, once the neighbors were eliminated using a preliminary PSF derived from the brightest, least contaminated $\sim 15$ stars. Both groups of PSF stars were interactively selected. We then used the ALLSTAR program to apply the resulting PSF to the identified stellar objects and to create a subtracted image which was used to find and measure magnitudes of additional fainter stars. This procedure was repeated three times for each frame. We examined the final subtracted images and corroborated that all the stars in the cluster field were eliminated. Finally, we standardized the resulting instrumental magnitudes and combined all the independent measurements using the stand-alone DAOMATCH and DAOMASTER programs, kindly provided by Peter Stetson. The final information consists of a running number per star, its $x$ and $y$ coordinates, the measured $V$ magnitudes and $B-V$ and $V-I$ colors, and the observational errors $\sigma(V), \sigma(B-V)$ and $\sigma(V-I)$. Only a portion of Table 1, which gives this information for a total of 1677 stars, is shown here for guidance regarding its form and content. The whole content of Table 1 is available in the online version of the journal. Fig. 1 shows the $V$ magnitude and $B-V$ and $V-I$ color errors as provided by DAOPHOT. 


\section{Color-Magnitude Diagram cleaning}

Fig. 2 depicts the $(B-V, V-I)$ diagram for all the measured stars and the $(V, B-I)$ Color-Magnitude Diagrams (CMDs) for those stars located inside or outside a circle of radius 160 pixels centered on the cluster. As can be figured out, this simple circular CMD extraction around the cluster center could lead to a wrong age, since this is obviously composed of stars of different stellar populations. Consequently, it is hardly possible to assess whether the whole extent of the observed populous Main Sequence (MS) or the subgiant and red giant branches (SGB,RGB) trace the fiducial cluster features.

With the aim of cleaning the cluster CMDs from the unavoidable star field

contamination we applied a procedure designed by Piatti \& Bica (2012) which makes use of variable cells in the CMDs. The cells are adjusted in such a way that they result bigger in CMD regions with a scarce number of field stars, and viceversa. This way, we reproduce the field CMD as closely as possible on the cluster CMD. The method does not need to know whether a star is placed close to the cluster center nor the cluster radial density profile to infer a membership probability. However, it takes into account the star field density, since the more populous a star field the larger the number of stars subtracted from the cluster CMD. As a result, the intrinsic spatial star distribution is uncovered within the cluster region. The method has shown to be able to eliminate stochastic effects in cluster CMDs caused by the presence of isolated bright stars, as well as, to make a finer cleaning in the most populous CMD regions.

For ESO 51-SC09 we cleaned a circular region centered on the cluster with a radius twice as big as the Full-Width at Half Maximum (FWHM) of its stellar density radial profile, as fitted by the IRAF NGAUSSFIT routine. As for the reference star fields, we used four different regions with the same area as for the cluster. These circular field areas were placed toward the NE, NW, SE, and SW of the cluster farther than three times the 
estimated cluster's FWHM. This was done in order to take into account variations in the spatial density, magnitudes, and colors of field stars. Thus, we obtained four different cleaned CMDs. When comparing those CMDs, one may find stars that have kept not subtracted in most of the times, while other stars were subtracted in most of the program executions. The different number of times that a star keeps not subtracted can then be converted into a measure of the probability of being a fiducial feature of the cluster field. Thus, we were able to distinguish stellar populations projected onto the cluster field that have a probability $\mathrm{P}<25 \%$ of being a genuine cluster population, i.e., a typical foreground population; stars that could indistinguishably belong to the star field or to ESO 51-SC09 (P $=50 \%)$; and stars that are predominantly found toward the cluster field $(\mathrm{P}>75 \%)$ rather than in the star field population. Fig. 3 shows the stars with chances of being a cluster feature higher than $75 \%$.

As can be seen, the cluster MS resulted successfully uncovered, especially its Turn Off (TO). In addition, some stars in the SGB and RGB, as well as red clump (RC) and blue and red horizontal branch stars also probably belong to the cluster stellar population. At a first glance, it appears that we are dealing with an old SMC cluster. Despite the presence of some interlopers and the absent of some cluster stars, this is the first time that a fiducial CMD is obtained for the cluster. From the cleaned cluster photometry, we fitted its stellar density radial profile with a Gaussian function and obtained an intrinsic FWHM of $(0.58 \pm$ $0.06)^{\prime}$, which converts into $(10 \pm 1)$ pc if a SMC distance of $60 \mathrm{kpc}$ is adopted (Glatt et al. 2010) for the cluster. On the other hand, the field stars observed along the line-of-sight toward ESO 51-SC09 appear to be composed by intermediate-age to old stars, although the dominant population is featured by a MS TO fainter (older) than that of the cluster $\left(\Delta V_{\mathrm{TO}}\right.$ $\sim 0.5 \mathrm{mag}$ ), by a noticeable populous SGB, and by a RGB more metal-poor than that of ESO 51-SC09, placed toward bluer colors. 


\section{Age and metallicity estimates}

The age was first calculated by determining the difference in $V$ magnitude between the RC and the MS TO from the cluster CMD (see Fig. 3) and then using the equation for the

Morphological Age Index (MAI Janes \& Phelps 1994) to obtain the age. As Janes \& Phelps (1994) showed, MAI is well correllated with the logarithm of cluster ages, as determined by fitting to theoretical isochrones. Note that this age measurement technique does not require absolute photometry and is independent of reddening as well. An additional advantage is that we do not need to go deep enough to see the extended cluster MS but only its MS TO. The derived $\delta V$ difference resulted to be $2.50 \pm 0.15 \mathrm{mag}$; its uncertainty $\sigma(\delta V)$ was estimated by considering the photometric errors at the RC and MS TO $V$ magnitudes (see Fig. 1) and the intrinsic dispersion in the CMD (Fig. 3). We adopted uncertainties five times larger than the photometric errors at the RC and MS TO $V$ magnitudes. The computed cluster age turned out to be $(8.3 \pm 1.6)$ Gyr. As can be seen, although the age error is slightly large, ESO 51-SC09 is clearly a new discovered relatively old/old cluster in the SMC.

Since Geisler et al. (1997) showed that $\delta V$ is very well-correlated with $\delta T_{1}$ (correlation coefficient $=0.993)$, we calculated the $\delta T_{1}$ index using their equation $(3)$, and then derived an age estimate from their equation (4). The $\delta T_{1}$ index has proven to be a powerful tool to derive ages for star clusters older than 1 Gyr, independently of their metallicities (Bica et al. 1998; Piatti et al. 2011). We obtained $\delta T_{1}=2.70 \pm 0.15$ mag, which in turn converts into an age of $(6.5 \pm 1.0)$ Gyr. This value is in excellent agreement within the quoted uncertainties with that derived from $\delta V$. Note that both luminosity differences were independently calibrated in terms of cluster ages.

We finally fitted theoretical isochrones to the cluster CMD to get an additional age estimate, by taking advantage of the theoretical isochrones calculated with core overshooting 
by Marigo et al. (2008). We adopted chemical compositions from $Z=0.001$ to 0.004 in steps of 0.0005 for the isochrone sets which cover the metallicity range of most of the SMC relatively old/old clusters studied in detail so far (Dias et al. 2010).

We then selected a set of isochrones in steps of $\Delta \log t=0.05$ and superimposed them on the cluster CMDs, once they were properly shifted by the corresponding $E(B-V)$ color excess and by the SMC apparent distance modulus. The estimation of the cluster reddening value was made by interpolating the extinction maps of Burstein \& Heiles (1982, hereafter $\mathrm{BH})$. They furnish us with a foreground $E(B-V)$ color excess equals to zero. Schlegel et al. (1998, hereafter SFD) obtained full-sky maps from 100- $\mu \mathrm{m}$ dust emission. We also used SFD's reddening maps to prove that when maximum and minimum extinction values are compared, the interstellar reddening is uniform across the observed field. We computed $E(B-V)_{S F D}$ color excesses for a grid in the $(l, \mathrm{~b})$ Galactic coordinate plane, with steps of $\Delta(l, \mathrm{~b})=\left(0.01^{\circ}, 0.01^{\circ}\right)$ covering the whole observed field. We obtained 25 color excess values for the cluster field. Then, for the resulting $E(B-V)_{S F D}$ values, we built a histogram and calculated its center and FWHM. Since the FWHM value turned out to be quite small, we assumed that the interstellar absorption is uniform across the cluster field. Finally, we adopted $E(B-V)=0.02 \pm 0.01$. As for the cluster distance modulus, we adopted the value of the SMC distance modulus $(m-M)_{o}=18.90 \pm 0.10$ recently reported by Glatt et al. (2010). Considering BH reddening values for populous SMC clusters, Crowl et al. (2001) found the line-of-sight depth of the galaxy to be approximately $6 \mathrm{kpc}$. Then, bearing in mind that ESO 51-SC09 could be placed in front of or behind the main body of the SMC, we concluded that the difference in apparent distance modulus could be as large as $\Delta\left(V-M_{V}\right) \sim 0.2 \mathrm{mag}$, if a value of $60 \mathrm{kpc}$ is adopted for the mean SMC distance. Given the fact that an uncertainty of 0.2-0.3 mag was estimated when adjusting the isochrones to the cluster CMD in magnitude, our simple assumption of adopting a unique value for the cluster distance modulus should not dominate the error budget in our final result. In fact, 
when over-plotting the Zero Age Main Sequence on the observed cluster CMD, previously shifted by the $E(B-V)=0.02$ and $(m-M)_{o}=18.90$, an excellent match was generally found, especially for the RC $V$ magnitude.

In the matching procedure, we used different isochrones for each metallicity level, ranging from slightly younger than the derived cluster age to slightly older. Finally, we adopted as the cluster age the one corresponding to the isochrone which best reproduced the cluster main features in the CMD. The presence of the RC, the SGB, the RGB in the cluster CMD made the fitting procedure easier, particularly in order to disentangle the cluster metallicity. The cluster age and metallicity resulted to be $t=(6.3 \pm 1.0)$ Gyr and $[\mathrm{Fe} / \mathrm{H}]=$ $(-1.00 \pm 0.15)$ dex, respectively, where the uncertainties come from different combinations of $(t,[\mathrm{Fe} / \mathrm{H}])$ pairs that reasonably adjust the observed dispersion in the cluster CMD. Fig. 3 shows the result of the fitting, where we plotted the isochrone of the adopted cluster age $(\log t=9.8)$ and two additional isochrones bracketing the derived age $(\log t=9.7,9.9)$ for $Z=0.002$. Finally, we averaged the three different age estimates and derived a mean age for ESO 51-SC09 of $(7.0 \pm 1.3)$ Gyr. This mean age places ESO 51-SC09 within the oldest known clusters of the SMC, only younger than NGC 121 (10.6 Gyr, Dolphin et al. 2001), HW 42 (9.3 Gyr, Piatti 2011b), NGC 361 (8.1 Gyr, Mighell et al. 1998), and Lindsay 1 (7.5 Gyr, Glatt et al. 2008). We used the $\delta V$ index and the isochrone fitting to the field star CMD to estimate a mean age and metallicity of $\sim 10-13$ Gyr and $-1.3 \pm 0.2$ dex, respectively, for the dominant star field projected toward the cluster direction. The remarkable different ages and metallicities of ESO 51-SC09 and the dominant field stellar population could be explained if we assume that the cluster was born in other part of the galaxy and, because of its orbial motion, it is observed at the current location. 


\section{Summary}

In this study we present for the first time CCD BVI photometry of stars in the field of the unstudied SMC cluster ESO 51-SC09. The data were obtained at the ESO NTT with the EMMI attached under high quality photometric conditions. We are confident that the photometric data yield accurate morphology and position of the main cluster features in the CMD. To disentangle the cluster features from those belonging to its surrounding field, we applied a subtraction procedure to statistically clean the cluster CMD from field star contamination. The method has shown to be able to eliminate stochastic effects in the cluster CMDs caused by the presence of isolated bright stars, as well as, to make a finer cleaning in the most populous CMD regions. The FWHM of the genuine cluster stellar density radial profile turned out to be $(0.58 \pm 0.06)^{\prime}$, which converts into $(10 \pm 1)$ pc if a SMC distance of $60 \mathrm{kpc}$ is adopted. Using the cleaned cluster $(V, B-V)$ diagram, we estimated its age and metallicity using the $\delta V$ and $\delta T_{1}$ indices and fitting theoretical isochrones. The three different age estimates are in excellent agreement, resulting in a mean value of $(7.0 \pm 1.3)$ Gyr. A metallicity of $[\mathrm{Fe} / \mathrm{H}]=(-1.00 \pm 0.15)$ dex was estimated from the fitting of theoretical isochrones to the cluster CMDs. We thus report that ESO 51-SC09 belongs to the group of the oldest SMC clusters, only younger (mean values) than NGC 121, HW 42, NGC 361, and Linday 1. The cluster is placed in a region of the SMC where probably it has not been born, since the mean age and metallicity of the dominant field stellar population is remarkable older (age 10-13 Gyr) and more metal poor $([\mathrm{FeH}]=$ $-1.3 \pm 0.2 \mathrm{dex})$. The cluster could reach its current location because of its orbital motion.

I greatly appreciate the suggestions raised by the reviewer which helped me to improve the manuscript. This work was partially supported by the Argentinian institutions CONICET and Agencia Nacional de Promoción Científica y Tecnológica (ANPCyT). 


\section{REFERENCES}

Bica E., Bonatto C., Dutra C.M., Santos Jr. J.F.C., 2008, MNRAS, 389, 678

Bica E., Geisler D., Dottori H., Piatti A.E., Clariá J.J., Santos Jr. J.F.C. 1998, AJ, 116, 723

Bonatto C., Bica E. 2010, MNRAS, 403, 996

Burstein D., Heiles C. 1982, AJ, 87, 1165 (BH)

Crowl H.H., Sarajedini A., Piatti A.E., Geisler D., Bica E., Clariá J.J., Santos Jr. J.F.C. 2001, AJ, 122, 220

Dias B., Coelho P., Barbuy B., Kerber L., Idiart T. 2010, A\&A, 520, A85

Dolphin A.E., Walker A.R., Hodge P.W., Mateo M., Olszewski E.W., Schommer R.A., Suntzeff N.B., 2001, ApJ, 562, 303

Geisler D., Bica E., Dottori H., Clariá J.J., Piatti A.E., Santos Jr., J.F.C., 1997, AJ, 114, 1920

Glatt K., Grebel E.K., Sabbi E., Gallagher J.S., Nota A., Sirianni M., Clementini G., Tosi M., Harbeck D., Koch A., et al. 2008, AJ, 136, 1703

Glatt K., Grebel E.K., Koch A. 2010, A\&A 517, 50

Janes K.A., Phelps R.L. 1994, AJ, 108, 1773

Jannuzi B.T., Claver J., Valdes F. 2003, The NOAO Deep Wide-Field Survey MOSAIC Data Reductions, http://www.noao.edu/noao/noaodeep/ReductionOpt/frames.html

Landolt A. 1992, AJ, 104, 340

Lauberts A. 1982, ESO/Uppsala Survey of the ESO (B) Atlas. European Southern Observatory, Garching bei Munchen 
Marigo P., Girardi L., Bressan A., Groenewegen M.A.T., Silva L., Granato G.L. 2008, A\&A, 482, 883

Mighell K.J., Sarajedini A., French R.S. 1998, AJ, 116, 2395 (MSF)

Piatti A.E., 2011b, MNRAS, 416, L89

Piatti A.E., 2011a, MNRAS Letters, 418, L69

Piatti A.E, Bica E. 2012, MNRAS, (in press, arXiv:1207.4762)

Piatti A.E., Clariá J.J., Bica E., Geisler D., Ahumada A.V., Girardi L. 2011, MNRAS, 417, 1559

Piatti A.E., Santos Jr. J.F.C., Clariá J.J., Bica E., Sarajedini A., Geisler D. 2001, MNRAS, 325,792

Schlegel D.J., Finkbeiner D.P., Davis M. 1998, ApJ, 500, 525 (SFD)

Stetson P.B., Davis L.E., Crabtree D.R., 1990, in ASP Conf. Ser. 8, CCDs in Astronomy (San Francisco: ASP), 289 


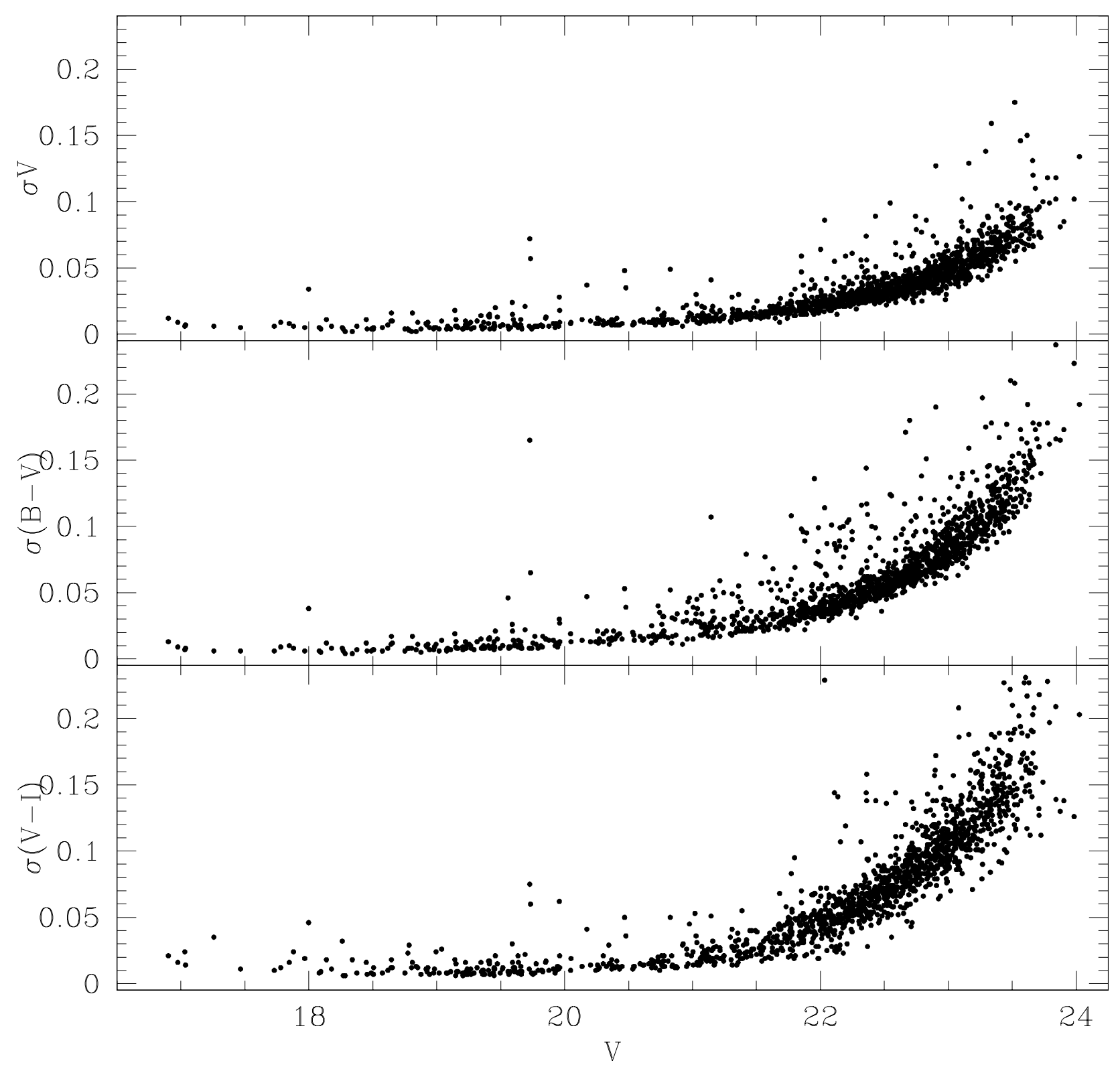

Fig. 1.- Magnitude and color photometric errors as a function of $V$ for the stars measured in the field of ESO 51-SC09. 


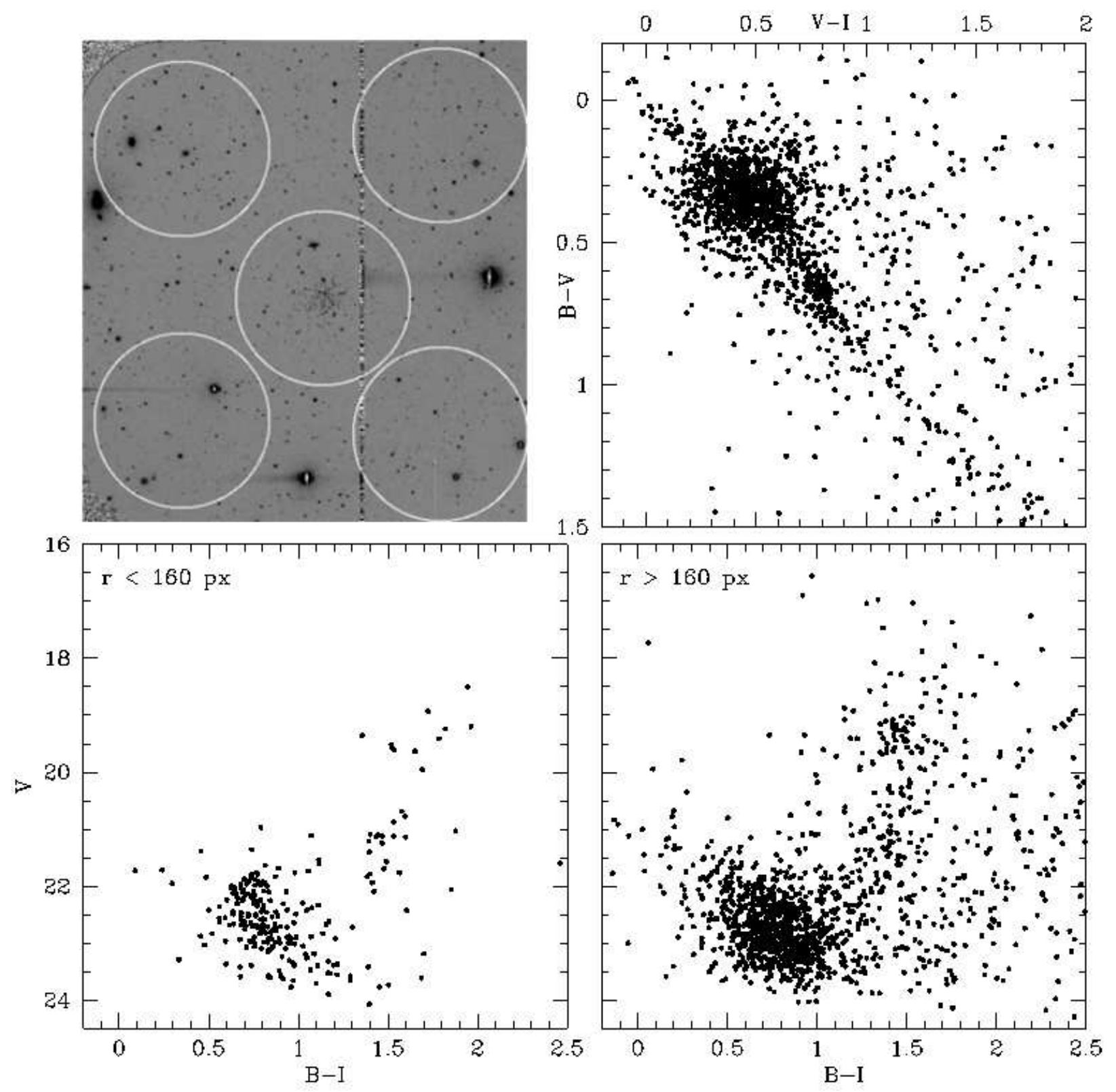

Fig. 2.- $V$ image with the cluster and four field circular regions over-plotted (upper left). The radius of each circle is 320 pixels. North is up and east is to the left. The $(B-V, V-I)$ (upper-right) and the $(V, B-I)$ diagrams (bottom) for all the measured stars is also shown. 
Table 1. BVI data of stars in the field of ESO 51-SC09.

\begin{tabular}{|c|c|c|c|c|c|c|c|c|}
\hline Star & $\begin{array}{c}x \\
\text { (pixel) }\end{array}$ & $\begin{array}{c}y \\
\text { (pixel) }\end{array}$ & $\begin{array}{c}V \\
\text { (mag) }\end{array}$ & $\begin{array}{l}\sigma(V) \\
(\mathrm{mag})\end{array}$ & $\begin{array}{l}B-V \\
(\mathrm{mag})\end{array}$ & $\begin{array}{c}\sigma(B-V) \\
(\mathrm{mag})\end{array}$ & $\begin{array}{l}V-I \\
(\mathrm{mag})\end{array}$ & $\begin{array}{c}\sigma(V-I) \\
(\mathrm{mag})\end{array}$ \\
\hline- & - & - & - & - & - & - & - & - \\
\hline 41 & 1168.79 & 156.49 & 20.345 & 0.009 & 0.752 & 0.015 & 0.866 & 0.029 \\
\hline 42 & 1491.06 & 156.82 & 18.262 & 0.005 & 1.525 & 0.008 & 2.136 & 0.032 \\
\hline 43 & 847.14 & 157.07 & 21.997 & 0.024 & 0.493 & 0.042 & 0.663 & 0.049 \\
\hline- & - & - & - & - & - & - & - & \\
\hline
\end{tabular}
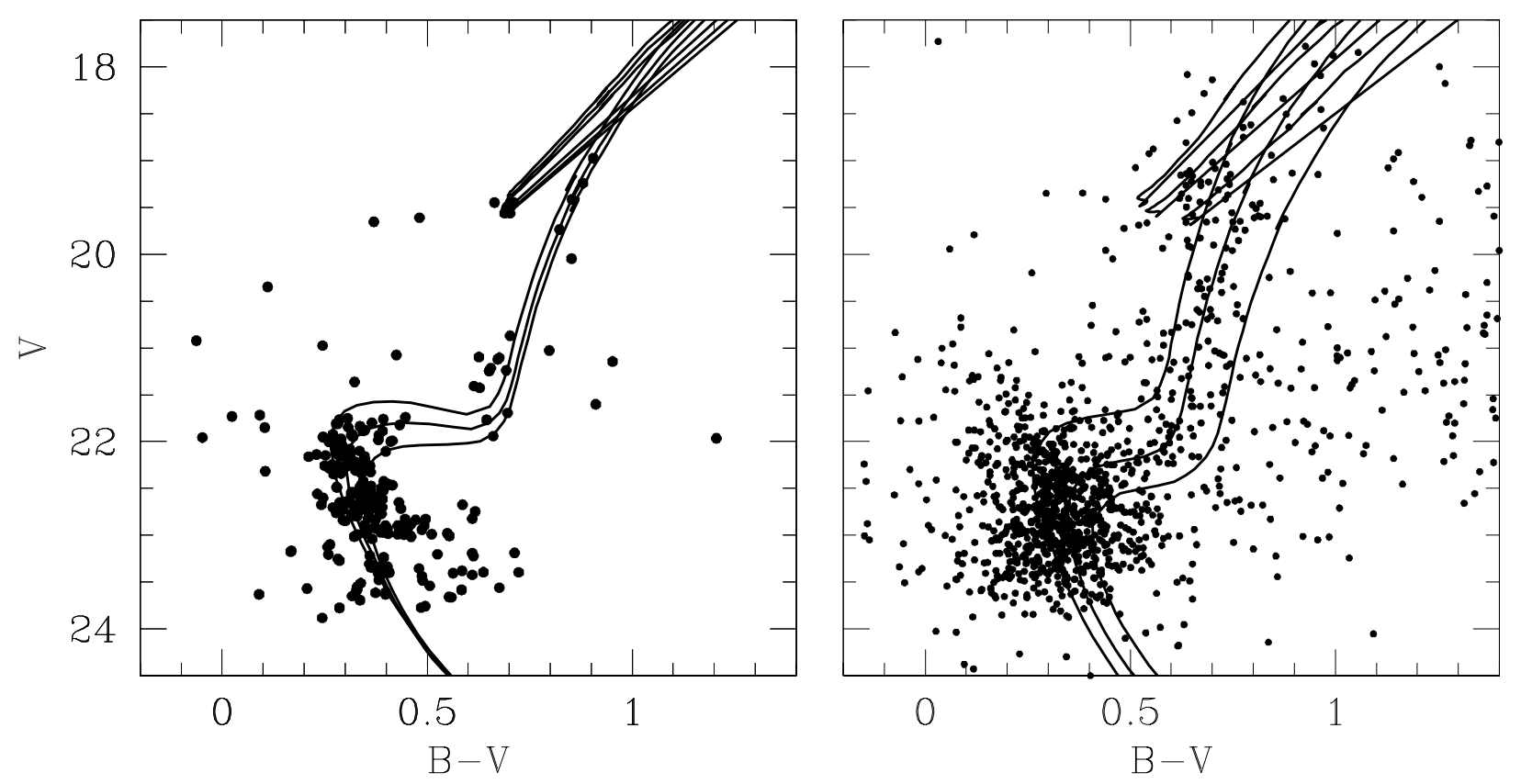

Fig. 3.- Left: CMD of ESO 51-SC09 with the Padova (Marigo et al. 2008) isochrones for $\log t=9.7,9.8$, and 9.9 , and $Z=0.002$. Right: Field CMD with isochrones for $\log t=9.9(\mathrm{Z}=$ $0.0005), 10.0(\mathrm{Z}=0.001)$, and $10.1(\mathrm{Z}=0.002)$. 